\title{
A LAGRANGIAN SOLUTION TO THE RELATIONSHIP BETWEEN A DISTRIBUTED SOURCE AND CONCENTRATION PROFILE
}

\author{
JON S. WARLAND ${ }^{\star}$ and GEORGE W. THURTELL \\ Dept. of Land Resource Science, University of Guelph, Guelph, Ontario, Canada N1G 2W1
}

(Received in final form 15 February 2000)

\begin{abstract}
A new solution is presented to the problem of relating source strength and concentration profiles within a plant canopy. The solution is based on the Lagrangian dispersion theory developed by G. I. Taylor in 1921. A dispersion matrix is derived that relates the source and concentration profiles based on profiles of the turbulent length and velocity scales. The matrix translates the effects of persistence (a temporal effect) into spatial coordinates and represents the change from near-field to far-field in a continuous fashion, successfully accounting for both regimes. A test of the new model using wind-tunnel data showed excellent quantitative agreement between model and measurements. A comparison was also made with M. R. Raupach's localized near-field theory, which underestimated the near-field effect in the wind-tunnel data and relative to the new model.
\end{abstract}

Keywords: Canopy modelling, Lagrangian theory, Micrometeorology, Turbulent transport.

\section{Introduction}

The relationship between a scalar source and its concentration profile within and just above a plant canopy has been an important problem of micrometeorology for many years. Its solution is important both for plant canopy modelling and for improved trace gas flux measurement. Micrometeorological flux measurement methods traditionally require large fetches and instrumentation well above a canopy. This limits micrometeorologists to flat, uniform terrain, and imposes large costs for working in tall canopies such as forests. Additionally, in areas of work with a vegetation component, such as land surface processes and crop growth modelling, knowledge of the in-canopy concentrations of water vapour and carbon dioxide is often important. To predict this one needs to know something of how the source strengths and concentration profiles are related. Therefore a solution to the in-canopy flux-profile problem would be very useful for a variety of applications ranging from improved trace gas exchange measurements to crop modelling.

Early theories of atmospheric dispersion developed the notion of an 'eddy diffusivity' (e.g., Taylor, 1915; Richardson, 1920). These theories postulated that turbulence acts on atmospheric scalars to disperse them in a diffusive manner. Attempts to apply this theory to plant canopies found, among other difficulties, the problem of counter-gradient fluxes, implying negative eddy diffusivities within

^ E-mail: jwarland@1rs.uoguelph.ca 
canopies. It is now commonly acknowledged that the failure of eddy diffusion theory within canopies is due to the effects of persistence caused by the correlated nature of turbulent movements (Raupach, 1987). In other words, when considering the ensemble-averaged dispersion of particles in a Lagrangian frame from the initial time when the particles are 'marked', the cloud initially disperses in a linear fashion, and at large travel times the cloud becomes diffusive. The initial dispersion regime is called the 'near-field' and the diffusive regime the 'far-field'.

The most common approach to solving this problem in the last two decades has been particle trajectory simulations based on random-walk modelling. Many of these models now exist, and have been summarized by Rodean (1996). However, successful application (e.g., Leclerc and Thurtell, 1990) relies on extensive calculations that are very demanding of computer time. Therefore, they are less than practical for daily use or for coupling with models of related processes. An alternative approach is to find an analytical model that captures the Lagrangian nature of turbulent dispersion. A noteworthy attempt at this is localized nearfield theory (LNF) (Raupach, 1989). In LNF, however, the near-field calculation assumes homogeneous dispersion; we believe that better results can be attained by accounting for the inhomogeneity of the dispersion when considering near-field effects.

Other investigations have focused on developing better insight into the physical structure of turbulence (e.g., Raupach et al., 1986, 1996; Collineau and Brunet, 1993a,b; Shaw et al., 1995). These studies are not only fascinating in their own right, but have provided valuable insight into the underlying physics of canopy transport processes. Of note in this work is the mixing-layer analogy put forward by Raupach and colleagues (Raupach et al., 1989, 1996), who showed that canopy flows bear much more resemblance to classic mixing-layer flows (see Wygnanski and Fiedler, 1970) than to surface-layer flows. However, this knowledge is not sufficient to predict canopy transport processes, since to do so also involves a description of the dissipation process itself.

Our paper reports on a new method of relating scalar concentration profiles to source distributions through a 'dispersion matrix'. The matrix is derived from Lagrangian dispersion theory and uses profiles of turbulence statistics to determine the dispersion. The matrix translates the effects of persistence (a temporal effect) into spatial coordinates and represents the change from near-field to far-field in a continuous fashion, successfully accounting for both regimes. The derivation, described first qualitatively and then mathematically in Section 2, is based on a homogeneous scenario and then assumes that dispersion in inhomogeneous turbulence behaves in a similar fashion. Tests of the model with wind-tunnel data, Section 4 , indicate that these assumptions provide a very reasonable approximation. Section 5 provides a quantitative comparison of the present model and LNF through the use of a 'near-field resistance' (Van Den Hurk and McNaughton, 1995; McNaughton and Van Den Hurk, 1995). This resistance term also offers 
one possible simplification of the model for use in models requiring a one-layer canopy description.

\section{Derivation of the Dispersion Matrix}

To visualize the derivation in a qualitative manner before developing the mathematics, consider a thin horizontal layer within a plant canopy. We can imagine this thin layer as a plane source of a scalar quantity, such as water vapour. The material released at any instant of time (which we will call a 'puff') will disperse up and down from the source in a Gaussian fashion (assuming homogeneous turbulence for simplicity), with a time-dependent concentration profile. At each instant, another puff is released, which also spreads in a similar fashion. Over time, the concentration profile that develops will be the sum of all the puffs released, each of which has a different travel time, and therefore a different distribution. This is illustrated in Figure 1, which shows several puffs released at various times from a plane at some height, along with their summed concentration profile. Over a long time, the concentration continues to increase, reaching infinity at infinite time. However, the gradient of concentration becomes constant and finite. Therefore, if we can determine the limiting value of the gradient of concentration, we then know the shape of the profile. Further, if we assume that the canopy is composed of several of these layers stacked on top of each other, we can then find the gradient at any height by taking the sum of the gradients produced by each layer. It is this conception that we will now proceed to develop mathematically. In order to do so, we begin with a Lagrangian analysis of turbulent dispersion, which describes the dispersion as a function of time. We then manipulate the equations to transform the temporal description of dispersion into spatial coordinates. This enables us to calculate the gradient created by the source layers, which allows us to draw the resulting profile.

Taylor (1921) found that the standard deviation of particle position for an ensemble average of marked particles dispersing in homogeneous, stationary turbulence was described for a given time $t$ by

$$
\sigma_{z}^{2}=2 \sigma_{w}^{2} \tau_{L}\left[t-\tau_{L}+\tau_{L} \exp \left(-t / \tau_{L}\right)\right]
$$

and assuming a correlation coefficient $R=\exp \left(-t / \tau_{L}\right)$. Here, $\sigma_{w}$ is the velocity scale of the particles (described by the standard deviation of vertical velocity for the one-dimensional case) and $\tau_{L}$ is the time scale of the motions of the particles - the time over which a particle's motion remains correlated with its previous movements.

Equation (1) has limits

$$
\lim _{t / \tau_{L} \rightarrow 0} \sigma_{z}^{2}=\sigma_{w}^{2} t^{2}
$$




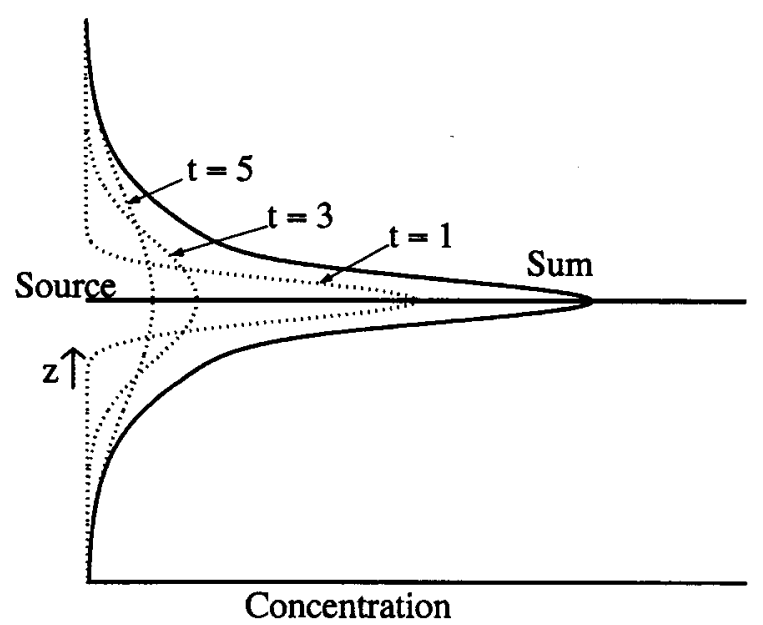

Figure 1. Illustration of continuous plane source. The source is at some height $z_{s}$ and the puffs that have dispersed for time $t$ add up to the total concentration profile labelled 'sum'.

$$
\lim _{t / \tau_{L} \rightarrow \infty} \sigma_{z}^{2}=2 \sigma_{w}^{2} \tau_{L} t
$$

known respectively as the near-field and far-field. For later discussion purposes we also define the region $t \sim \tau$ as the mid-field. These limits show that dispersion proceeds first as a linear function of time, independent of the time scale, and later as the square root of time and dependent on the time scale. Note that in this homogeneous, stationary scenario the time scale is constant in both space and time. These near-field and far-field regimes were shown graphically by Richardson (1920) in long-exposure photographs of smoke plumes.

To derive the present model, we first consider dispersion in homogeneous, stationary turbulence from an instantaneous plane source $Q$. A plane source is chosen as a simple idealization of an extensive canopy. This is described by the Gaussian distribution (Csanady, 1973)

$$
C=\frac{Q}{\sqrt{2 \pi \sigma_{z}^{2}}} \exp \left[\frac{-\left(z-z_{s}\right)^{2}}{2 \sigma_{z}^{2}}\right]
$$

where $z_{s}$ is the height of the source $Q\left[\mathrm{M} \mathrm{L}^{-2}\right]$ (or $\mathrm{J} \mathrm{m}^{-2}$ for the case of an energy, as opposed to mass, source). $\sigma_{z}$ in this equation is described by Equation (1) for the correlated condition specified above. Equation (3) requires $C=\infty$ at $t=0$, which is due to the fact that $Q$ occupies zero volume at $t=0$.

For a continuous source $q\left[\mathrm{M} \mathrm{L}^{-2} \mathrm{~T}^{-1}\right]$ (or $\mathrm{W} \mathrm{m} \mathrm{m}^{-2}$ for an energy source) each interval $d t$ will produce source $q d t$, which will change the concentration by amount $d c$. This allows us to re-write Equation (3) as

$$
\frac{\partial c}{\partial t}=\frac{q}{\sqrt{2 \pi \sigma_{z}^{2}}} \exp \left[\frac{-\left(z-z_{s}\right)^{2}}{2 \sigma_{z}^{2}}\right] .
$$


This equation integrates to infinity, however the gradient of concentration with respect to $z$ becomes constant at large $t$, representing the one-dimensional equilibrium state of the idealized canopy. Taking the derivative of Equation (4) with respect to space gives

$$
\frac{\partial}{\partial z}\left(\frac{\partial c}{\partial t}\right)=\frac{-q}{\sqrt{2 \pi \sigma_{z}^{2}}} \frac{\left(z-z_{s}\right)}{\sigma_{z}^{2}} \exp \left(\frac{-\left(z-z_{s}\right)^{2}}{2 \sigma_{z}^{2}}\right) .
$$

This can be interpreted physically as the contribution to the gradient at $z$ and $t$ of puff $q d t$. Using the generally valid assumption that

$$
\frac{\partial}{\partial z}\left(\frac{\partial c}{\partial t}\right)=\frac{\partial}{\partial t}\left(\frac{\partial c}{\partial z}\right)
$$

the gradient is

$$
\frac{\partial c}{\partial z}=\int_{0}^{\infty} \frac{-q}{\sqrt{2 \pi \sigma_{z}^{2}}} \frac{\left(z-z_{s}\right)}{\sigma_{z}^{2}} \exp \left(\frac{-\left(z-z_{s}\right)^{2}}{2 \sigma_{z}^{2}}\right) \partial t .
$$

Equation (6) can be integrated with the near- and far-field limits of $\sigma_{z}$ (Equations 2) to give

$$
\begin{aligned}
& \left.\frac{\partial c}{\partial z}\right|_{N F}=\frac{-q}{\sqrt{2 \pi} \sigma_{w}\left|z-z_{s}\right|} \quad \text { as } z / L_{L} \rightarrow 0 \\
& \left.\frac{\partial c}{\partial z}\right|_{F F}=\frac{-q}{2 \sigma_{w} L_{L}} \quad \text { as } z / L_{L} \rightarrow \infty,
\end{aligned}
$$

where we have substituted the Lagrangian length scale $L_{L}=\sigma_{w} \tau_{L}$. The integration removes the time dependence, and the substitution of the length scale for the time scale completes the transformation to purely spatial coordinates. Note that $q$ is still a plane source (finite substance produced in zero volume), and so Equation (7a) states that an infinite gradient exists at $z=z_{s}$. This will be discussed in more detail below. Also note that the sign convention in Equations (7) implies that the gradient of interest is above the source; for gradients beneath the source Equations (7) must be multiplied by -1 . This also explains the need for the absolute value in the near-field limit.

Using Equation (7a) we define near- and far-field dispersion coefficients from the general relationship $\partial c / \partial z=M q$ as

$$
M_{N F}= \begin{cases}\frac{-1}{\sqrt{2 \pi} \sigma_{w}\left(z-z_{s}\right)} & \text { for } z>z_{s} \\ \frac{1}{\sqrt{2 \pi} \sigma_{w}\left(z_{s}-z\right)} & \text { for } z<z_{s},\end{cases}
$$




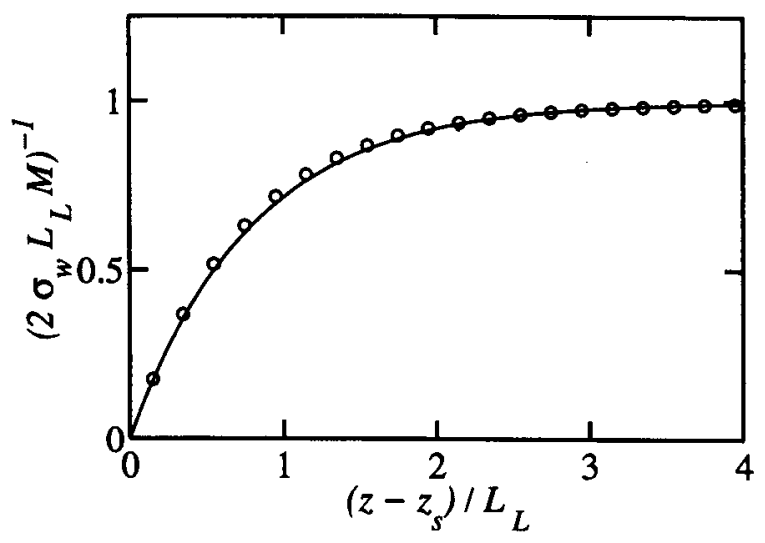

Figure $2 a$. Normalized inverse dispersion factor from the numerical integration of Equation (5) using full $\sigma_{z}$ from Equation (1) (circles) with normalized $M^{-1}$ from Equation (9) (line).

$$
M_{F F}=\left\{\begin{array}{l}
\frac{-1}{2 \sigma_{w} L_{L}} \text { for } z>z_{s} \\
\frac{1}{2 \sigma_{w} L_{L}} \text { for } z<z_{s},
\end{array}\right.
$$

where the sign convention accounts for the relative position of source and gradient heights and we have left the dispersion coefficient undefined at $z=z_{s}$ for the moment. To describe dispersion continuously from near- to far-field we propose the dispersion coefficient

$$
M= \begin{cases}\frac{-1}{2 \sigma_{w} L_{L}\left[1-\exp \left(-\sqrt{\frac{\pi}{2}} \frac{\left(z-z_{s}\right)}{L_{L}}\right)\right]} & \text { for } z>z_{s} \\ \frac{1}{2 \sigma_{w} L_{L}\left[1-\exp \left(-\sqrt{\frac{\pi}{2}} \frac{\left(z_{s}-z\right)}{L_{L}}\right)\right]} & \text { for } z<z_{s},\end{cases}
$$

which is equal to Equations (8) in the near- and far-field limits. To test the mid-field of this expression the inverse dispersion factor was calculated from a numerical integration performed on Equation (5) using the complete $\sigma_{z}$ expression from Equation (1). The normalized results are illustrated in Figures $2 \mathrm{a}$ and $2 \mathrm{~b}$, which show $\left(2 \sigma_{w} L_{L} M\right)^{-1}$ as a function of $\left(z-z_{s}\right) / L_{L}$. From these graphs it can readily be seen that Equation (9) is indeed a close approximation of the continuous nearfield/far-field dispersion described by Equation (1). The maximum error is 3.6\% at $\left(z-z_{s}\right) / L_{L}=0.5$.

The dispersion coefficient defined by Equation (9) is a continuous function for all $z \neq z_{s}$ describing the relationship between the gradient of concentration at every $z \neq z_{s}$ in relation to a continuous plane source $q$. In reality, we do not 


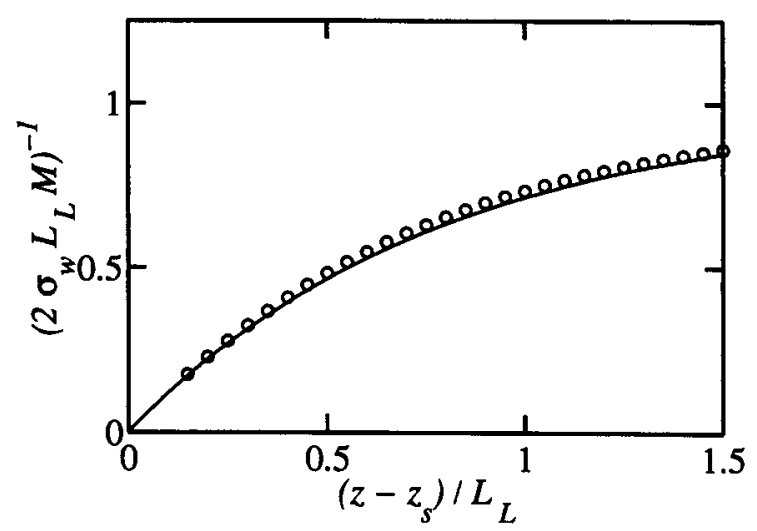

Figure $2 b$. Mid-field detail of inverse dispersion factor from numerical integration of Equation (5) using full $\sigma_{z}$ from Equation (1) (circles) with normalized $M^{-1}$ from Equation (9) (line).

experience plane sources and infinite concentrations. Further, we generally express concentrations and gradients at points that we assume to be representative of layers. Therefore, we wish to re-write the dispersion coefficient in terms of layers, as shown in Figure 3. Each layer is a slab of air of thickness $\Delta z$, at height specified by its mid-point $z_{i}$ with mean concentration $c_{i}$ and mean gradient across the layer $d c /\left.d z\right|_{i}$. The source $q$ is assumed (for the moment) to be distributed evenly across layer $z_{s}$, and so should be written $q \Delta z$ (where $q$ now has dimensions $\left[\mathrm{M} \mathrm{L}^{-3} \mathrm{~T}^{-1}\right]$ ). We can now write the dispersion coefficient as

$$
M=\left\{\begin{array}{cc}
\frac{-1}{2 \sigma_{w} L_{L}\left[1-\exp \left(-\sqrt{\frac{\pi}{2}} \frac{\left(z_{i}-z_{s}\right)}{L_{L}}\right)\right]} & \text { for } z_{i}>z_{s} \\
0 & \text { for } z_{i}=z_{s} \\
\frac{1}{2 \sigma_{w} L_{L}\left[1-\exp \left(-\sqrt{\frac{\pi}{2}} \frac{\left(z_{s}-z_{i}\right)}{L_{L}}\right)\right]} & \text { for } z_{i}<z_{s},
\end{array}\right.
$$

where $d c /\left.d z\right|_{i}=M_{i} q$ and we have set $M=0$ at $z_{i}=z_{s}$ for the time being in order to prevent a physically impossible infinite gradient. This problem of infinite gradients as $z_{i} \rightarrow z_{s}$ will be discussed below. To calculate the concentration profile, the concentration must be specified at one height, and the other points in the profile are given by

$$
c_{i+1}=c_{i}+\frac{1}{2}\left(\left.\frac{d c}{d z}\right|_{i}+\left.\frac{d c}{d z}\right|_{i+1}\right)\left(z_{i+1}-z_{i}\right) .
$$

To apply this model to 'real-world' scenarios, a boundary condition for the surface $(z=0)$ is required. For this we follow traditional dispersion modelling and 


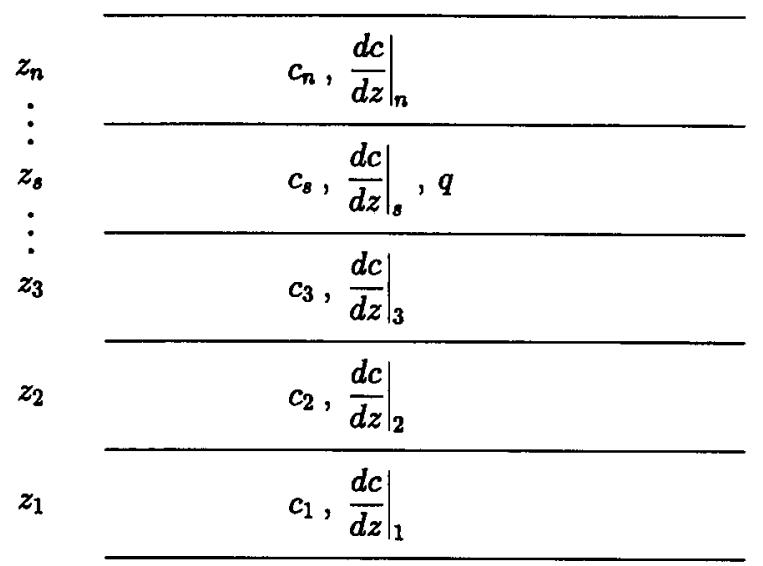

Figure 3. Schematic diagram of layers, where each layer has mean concentration $c_{i}$ and mean gradient $d c /\left.d z\right|_{i}$ with height specified by mid-point $z_{i}$. The source layer contains the distributed source $q$.

specify an image source to create a reflecting boundary (e.g., Csanady, 1973). This does not preclude a source term at the surface, corresponding to a surface source. The image source can be thought of as the probability of a parcel dispersing from the source to the surface and back up to the height of interest. The image source is added to the original source, and its dispersion coefficient is specified by the distance $z+z_{s}$ rather than the difference $z-z_{s}$. Thus the dispersion coefficient can now be re-written to include the reflecting boundary condition as

$$
M= \begin{cases}\frac{-1}{2 \sigma_{w} L_{L}\left[1-\exp \left(-\sqrt{\frac{\pi}{2}} \frac{\left(z_{i}-z_{s}\right)}{L_{L}}\right)\right]}- & \text { for } z_{i}>z_{s} \\ \frac{-1}{2 \sigma_{w} L_{L}\left[1-\exp \left(-\sqrt{\frac{\pi}{2}} \frac{\left(z_{i}+z_{s}\right)}{L_{L}}\right)\right]} & \text { for } z_{i}=z_{s} \\ \frac{1}{2 \sigma_{w} L_{L}\left[1-\exp \left(-\sqrt{\frac{\pi}{2}} \frac{\left(z_{i}+z_{s}\right)}{L_{L}}\right)\right]} & \\ \frac{1}{2 \sigma_{w} L_{L}\left[1-\exp \left(-\sqrt{\frac{\pi}{2}} \frac{\left(z_{s}-z_{i}\right)}{L_{L}}\right)\right]}- & \text { for } z_{i}<z_{s} .\end{cases}
$$

The contribution to the gradient in the source layer due to the source is still 0 , however the reflecting boundary implies that there will be a non-zero mean gradient in that layer. 
A distributed source is required to realistically model a canopy, necessitating sources specified in multiple layers. The gradient in one layer is now equal to the sum of the gradients created by each source layer. Mathematically,

$$
\left.\frac{d c}{d z}\right|_{i}=\mathbf{M}_{i j} q_{j}
$$

where the indicated matrix operation is a summation over the repeated index and the dispersion coefficient is now a matrix of the form

$$
\mathbf{M}_{i j}= \begin{cases}\frac{-1}{2 \sigma_{w} L_{L}\left[1-\exp \left(-\sqrt{\frac{\pi}{2}} \frac{\left(z_{i}-z_{j}\right)}{L_{L}}\right)\right]}- & \\ \frac{1}{2 \sigma_{w} L_{L}\left[1-\exp \left(-\sqrt{\frac{\pi}{2}} \frac{\left(z_{i}+z_{j}\right)}{L_{L}}\right)\right]} & \text { for } z_{i}>z_{j} \\ \frac{-1}{2 \sigma_{w} L_{L}\left[1-\exp \left(-\sqrt{\frac{\pi}{2}} \frac{\left(z_{i}+z_{j}\right)}{L_{L}}\right)\right]} & \text { for } z_{i}=z_{j} \\ \frac{1}{2 \sigma_{w} L_{L}\left[1-\exp \left(-\sqrt{\frac{\pi}{2}} \frac{\left(z_{j}-z_{i}\right)}{L_{L}}\right)\right]}- & \\ \frac{1}{2 \sigma_{w} L_{L}\left[1-\exp \left(-\sqrt{\frac{\pi}{2}} \frac{\left(z_{i}+z_{j}\right)}{L_{L}}\right)\right]} & \text { for } z_{i}<z_{j} .\end{cases}
$$

The dispersion coefficient is now written in bold face as a reminder that it is a matrix quantity.

In the preceding, we made the assumption that the contribution to the gradient at a height $z_{j}$ from the source at that height $q_{j}$ was zero. This is a valid assumption, and avoids a dispersion coefficient of infinity, however we still face the theoretical problem that this model calculates very large gradients near the source when the grid spacing is made very small. This can be seen in the homogeneous dispersion coefficient, which suffers the limit

$$
\lim _{\left(z-z_{s}\right) \rightarrow 0} \frac{1}{2 \sigma_{w} L_{L}\left[1-\exp \left(-\sqrt{\frac{\pi}{2}} \frac{z-z_{s}}{L_{L}}\right)\right]}=\infty .
$$

This happens because the near-field gradient has the same limit, i.e.

$$
\lim _{\left(z-z_{s}\right) \rightarrow 0} \frac{-q}{\sqrt{2 \pi} \sigma_{w}\left|z-z_{s}\right|}=\infty .
$$

This is due to the assumption of a plane source at $t=0$, which, when translated to spatial terms, becomes a plane source at $z=z_{s}$. As we consider smaller and 
smaller grids, or points very close to a source, we are in effect 'squeezing' the source into a plane source.

If we specify that the source must have an initial spatial distribution, we avoid forcing it into a plane. We can do this by assuming some initial distribution by specifying an 'offset' time $t_{0}$ so that

$$
\lim _{t / t_{L} \rightarrow 0} \sigma_{z}^{2}=\sigma_{w}^{2}\left(t+t_{0}\right)^{2} .
$$

This, in effect, specifies that the source is distributed about $z_{s}$ in Gaussian fashion with standard deviation $\sigma_{s}=\sigma_{w} t_{0}$. When we now integrate Equation (5) in the near-field (integrating still from 0 to $\infty$, but with the variable of integration now $\left.\left(t+t_{0}\right)\right)$, the gradient becomes

$$
\left.\frac{\partial c}{\partial z}\right|_{N F}=\frac{q}{\sqrt{2 \pi} \sigma_{w}\left|z-z_{s}\right|}\left[1-\exp \left(\frac{-\left(z-z_{s}\right)^{2}}{2 \sigma_{w}^{2} t_{0}^{2}}\right)\right] .
$$

The limiting value of this expression is

$$
\left.\lim _{\left(z-z_{s}\right) \rightarrow 0} \frac{\partial c}{\partial z}\right|_{N F}=\frac{q\left(z-z_{s}\right)}{\sqrt{8 \pi} \sigma_{w}^{2} t_{0}^{2}}
$$

We now must ask what the appropriate value of $\sigma_{s}$ is. By making it arbitrarily small relative to $z-z_{s}$ the exponential disappears. However, this is not satisfying because the original question was what happens when $z-z_{s}$ becomes arbitrarily small. For the case when our source is contained within one layer of the model, as in the wind-tunnel example discussed below, $\sigma_{s}$ should be specified so as to contain all the source $q$. For the case of $99.9 \%$ of $q$ within a source layer of thickness $\Delta z$, $\sigma_{s} \approx 0.16 \Delta z$ and the exponential term in Equation (18) is of the order $10^{-9}$ in the adjacent grid. 
On the other hand we can describe each layer with $\sigma_{s}=\Delta z$ so that the integral of the source layers equals the expected smooth profile of $q$. This proposition means that the dispersion matrix must be re-written as,

$$
\mathbf{M}_{i j}=\left\{\begin{array}{cc}
\frac{-\left[1-\exp \left(\frac{-\left(z_{i}-z_{j}\right)^{2}}{2 \Delta z_{j}^{2}}\right)\right]}{2 \sigma_{w} L_{L}\left[1-\exp \left(-\sqrt{\frac{\pi}{2}} \frac{\left(z_{i}-z_{j}\right)}{L_{L}}\right)\right]}- & \\
\frac{\left[1-\exp \left(\frac{-\left(z_{i}+z_{j}\right)^{2}}{2 \Delta z_{j}^{2}}\right)\right]}{2 \sigma_{w} L_{L}\left[1-\exp \left(-\sqrt{\frac{\pi}{2}} \frac{\left(z_{i}+z_{j}\right)}{L_{L}}\right)\right]} & \text { for } z_{i}>z_{j} \\
\frac{-\left[1-\exp \left(\frac{-\left(z_{i}+z_{j}\right)^{2}}{2 \Delta z_{j}^{2}}\right)\right]}{2 \sigma_{w} L_{L}\left[1-\exp \left(-\sqrt{\frac{\pi}{2}} \frac{\left(z_{i}+z_{j}\right)}{L_{L}}\right)\right]} & \text { for } z_{i}=z_{j} \\
\frac{\left[1-\exp \left(\frac{-\left(z_{i}-z_{j}\right)^{2}}{2 \Delta z_{j}^{2}}\right)\right]}{2 \sigma_{w} L_{L}\left[1-\exp \left(-\sqrt{\frac{\pi}{2}} \frac{\left(z_{j}-z_{i}\right)}{L_{L}}\right)\right]}- & \\
\frac{\left[1-\exp \left(\frac{-\left(z_{i}+z_{j}\right)^{2}}{2 \Delta z_{j}^{2}}\right)\right]}{2 \sigma_{w} L_{L}\left[1-\exp \left(-\sqrt{\frac{\pi}{2}} \frac{\left(z_{i}+z_{j}\right)}{L_{L}}\right)\right]} & \text { for } z_{i}<z_{j},
\end{array}\right.
$$

where $\Delta z_{j}$ is the thickness of the source layer $j$. Normalized results with and without this correction, along with LNF results, are shown in Figure 4 using the idealized profiles described below by Equations (27). As can be seen, the correction is small, but nonetheless important for avoiding physically absurd gradients near the source.

The final step in developing a realistic model is to apply it to the inhomogeneous turbulence found in the 'real-world'. Raupach (1987) has shown that canopy dispersion can be reasonably approximated using a homogeneous description of the turbulence. If, following this logic, we assume that inhomogeneous turbulence behaves similarly to homogeneous turbulence, then we can postulate that Equations (13) and (14) apply to the inhomogeneous case with $\sigma_{w}$ and $L_{L}$ replaced by their 


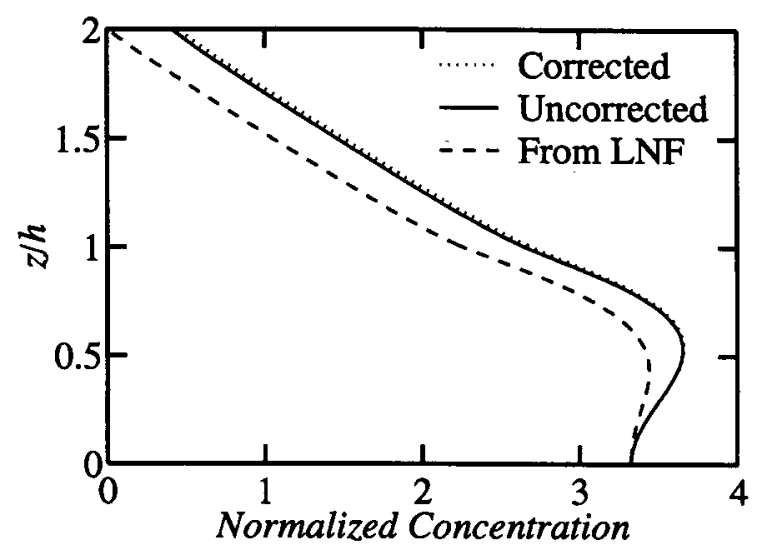

Figure 4. Normalized concentration profiles calculated using idealized profiles of Equation (27a) with $c_{0}=0.25, p=4$ and $q=2$. The present model shown with and without the correction for infinite plane sources. Results from LNF are also shown.

values for each layer, i.e., $\sigma_{w i}$ and $L_{L i}$. This leads to the final form of the dispersion matrix

$$
\mathbf{M}_{i j}=\left\{\begin{array}{cc}
\frac{-\left[1-\exp \left(\frac{-\left(z_{i}-z_{j}\right)^{2}}{2 \Delta z_{j}^{2}}\right)\right]}{2 \sigma_{w i} L_{L i}\left[1-\exp \left(-\sqrt{\frac{\pi}{2}} \frac{\left(z_{i}-z_{j}\right)}{\left(L_{L i}+L_{L j}\right) / 2}\right)\right]}- \\
\frac{\left[1-\exp \left(\frac{-\left(z_{i}+z_{j}\right)^{2}}{2 \Delta z_{j}^{2}}\right)\right]}{2 \sigma_{w i} L_{L i}\left[1-\exp \left(-\sqrt{\frac{\pi}{2}} \frac{\left(z_{i}+z_{j}\right)}{\left(L_{L i}+L_{L j}\right) / 2}\right)\right]} & \text { for } z_{i}>z_{j} \\
\frac{-\left[1-\exp \left(\frac{-\left(z_{i}+z_{j}\right)^{2}}{2 \Delta z_{j}^{2}}\right)\right]}{2 \sigma_{w i} L_{L i}\left[1-\exp \left(-\sqrt{\frac{\pi}{2}} \frac{\left(z_{i}+z_{j}\right)}{\left(L_{L i}+L_{L j}\right) / 2}\right)\right]} & \text { for } z_{i}=z_{j}(21) \\
\frac{\left[1-\exp \left(\frac{-\left(z_{i}-z_{j}\right)^{2}}{2 \Delta z_{j}^{2}}\right)\right]}{2 \sigma_{w i} L_{L i}\left[1-\exp \left(-\sqrt{\frac{\pi}{2}} \frac{\left(z_{j}-z_{i}\right)}{\left(L_{L i}+L_{L j}\right) / 2}\right)\right]}- & \\
\frac{\left[1-\exp \left(\frac{-\left(z_{i}+z_{j}\right)^{2}}{2 \Delta z_{j}^{2}}\right)\right]}{2 \sigma_{w i} L_{L i}\left[1-\exp \left(-\sqrt{\frac{\pi}{2}} \frac{\left(z_{i}+z_{j}\right)}{\left(L_{L i}+L_{L j}\right) / 2}\right)\right]} & \text { for } z_{i}<z_{j},
\end{array}\right.
$$

where the mean value of the two length scales is used in the exponent because dispersion occurs through exchange between the two layers, thus requiring the use 


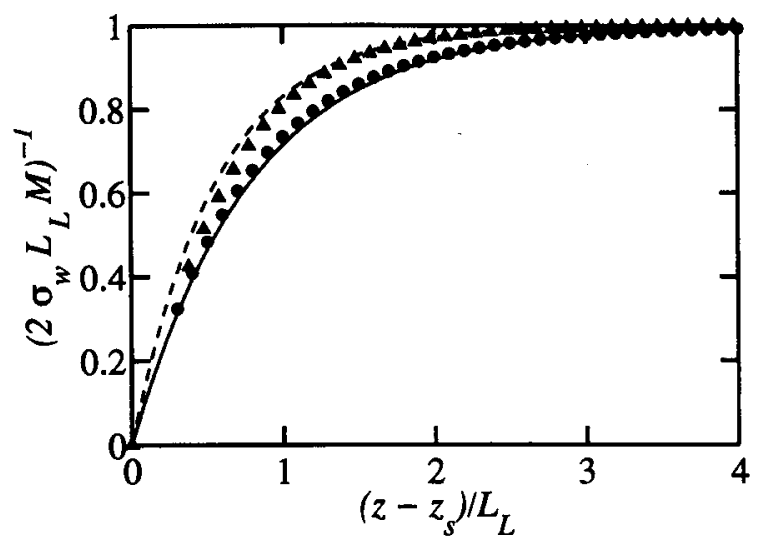

Figure 5. Comparison of alternative formulations $M$ and $M_{a}$. Equation (9) (solid line) and a numerical integration of Equation (5) using $\sigma_{z}$ from Equation (1) (circles). Equation (23) (dashed line) and a numerical integration of Equation (5) using $\sigma_{z}$ derived from $R=\exp \left(-\frac{\pi}{4} \frac{t^{2}}{\tau^{2}}\right)$ (triangles).

of both length scales in determining the extent of the near-field effect. Therefore, the final form of this model consists of Equations (11), (13) and (21).

\section{Alternative Formulations of $R(t)$}

To test the dependence of this model on the formulation of the correlation coefficient $R(t)$, the above derivation was repeated using an alternative suggested by Frenkiel (1953)

$$
R=\exp \left(-\frac{\pi}{4} \frac{t^{2}}{\tau^{2}}\right)
$$

Equation (22) was proposed to ensure a continuous tangent at the origin, thereby avoiding the implied infinite acceleration at $t=0$ with $R=\exp (-t / \tau)$, while maintaining the same area under the curve.

Following the steps outlined above leads to

$$
M_{a}=\frac{1}{2 \sigma_{w} L_{L}\left[1-\exp \left(-\sqrt{\pi} \frac{z-z_{s}}{L_{L}}\right)\right]},
$$

analogous to Equation (9) with the only difference being a factor of $\sqrt{2}$ in the exponent. Comparison of $M$ and $M_{a}$ is shown in Figure 5.

From Figure 5 it can be seen that the two correlation coefficients lead to very similar dispersion factors, although the alternative near-field slope is steeper and the far-field is reached sooner. This reduces the effect of the near-field in the model output. Tests of the model, discussed below, showed better agreement between 
data and the model with the original correlation function. This is indicative of the importance of the near-field effects, and commends the continued use of $R=\exp (-t / \tau)$ despite the discontinuity at the origin of the correlation curve.

\section{Comparison of the Model with Data}

Coppin et al. (1986) (hereafter CRL86) provide one of the few studies with detailed profiles of turbulence measurements within a canopy with a known source strength. This experiment used the Pye Laboratory wind tunnel at CSIRO, Australia to observe turbulence and turbulent transport within and above an artificial canopy. The canopy consisted of metal strips $6 \mathrm{~cm}$ tall and an elevated plane-equivalent source of heat at $4.8 \mathrm{~cm}$ served as a passive scalar. The source strength was $275 \mathrm{~W} \mathrm{~m}^{-2}$, and the insulated floor provided an almost ideal reflecting surface, as seen in CRL86's flux measurements. Turbulence measurements for the artificial canopy were reported by Raupach et al. (1986).

Since no known technique exists for the direct determination of the Lagrangian length scale, the measured Eulerian vertical length scale

$$
L_{E w}=\frac{\bar{u}}{\sigma_{w}^{2}} \int_{0}^{\tau_{0}} \overline{w^{\prime}(t) w^{\prime}(t+\tau)} d \tau
$$

was substituted. This assumption follows from Corrsin (1963) and Kraichnan (1964) who provided theoretical evidence that $L_{L} / L_{E} \sim 1$. Values of $\sigma_{w}$ and $L_{E w}$ were taken from Raupach et al. (1986) and input into the model along with the source strength. These same data were also used as input for the LNF calculations. The model produces a profile of gradients so the measured temperature nearest the surface was used to calculate the temperature profile. The results of this are plotted in Figure 6, along with CRL86's measured temperature profile.

For comparison, Figure 6 also shows the same calculation using LNF. Both models have the same far-field limit so the two model slopes agree above the canopy where the far-field regime dominates. However, within the canopy, where near-field effects are prominent due to the proximity of the source, LNF achieves less agreement with the data. This is due to LNF underestimating the near-field effect in this scenario, leading to an underestimation of the heat concentration near the source.

The present model shows excellent overall agreement with the wind-tunnel measurements, particularly within the canopy. There is some disagreement in the region around $z / h \approx 2$, which could be due to a number of factors. The far-field, as discussed above, is more dependent on the length scale than the near-field. Thus errors may be introduced by using the Eulerian length scale. Because each point is calculated as the cumulative sum of the gradients below it, an error in one point could affect all the points above it. To examine this, Figure 7 shows finite difference gradients from the model along with the measured gradients. Excellent agreement 


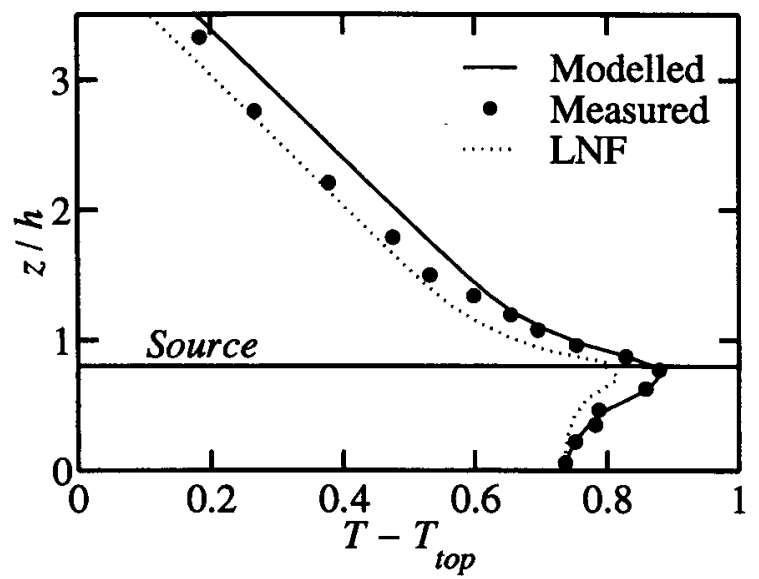

Figure 6. Comparison of CRL86's wind-tunnel data and the present model using Eulerian scales in place of the Lagrangian. Results from localized near-field theory also shown. Data are shown as the difference from the highest measured point $T_{t o p}$ at $z / h \approx 6.3$.

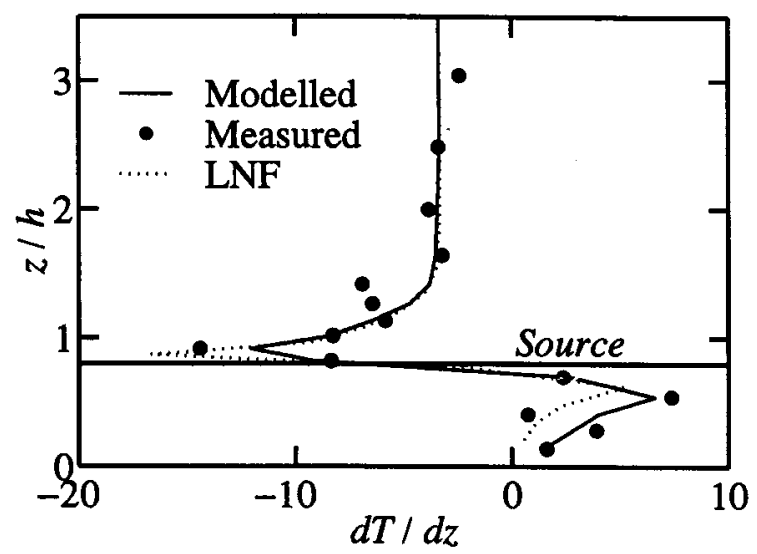

Figure 7. Gradients calculated by the model shown with gradients measured by CRL86. Results from localized near-field theory also shown.

is shown everywhere except around $z / h=1.3$. It is above here that the measured and modelled profiles disagree, though the modelled gradients above $z / h=1.3$ agree again with the measured. The measured points here, at $z / h=1.25$ and 1.4 , indicate an increasing gradient with height above the source, which is not physically likely, suggesting that the discrepancy between model and measurement may be due to errors in the data.

Results from LNF are also shown in Figure 7. The two models coincide where far-field effects dominate, but again the near-field is not fully captured by LNF. This confirms the objection to LNF that it underestimates the near-field effect in this canopy. 


\section{The Near-Field Resistance}

In two recent papers Van Den Hurk and McNaughton (1995) and McNaughton and Van Den Hurk (1995) derived a 'near-field resistance' $r_{n}$ to account for near-field effects within a simple two-layer canopy model. Their work was based on LNF using the near-field concentration term. The near-field resistance is defined as

$$
r_{n} \equiv \frac{\bar{C}_{n}}{F_{h}}
$$

where $F_{h}$ is the scalar flux density from the canopy and $\bar{C}_{n}$ is a mean near-field concentration calculated as

$$
\bar{C}_{n}=\int_{0}^{h} \phi(z) C_{n}(z) d z
$$

where $\phi(z)$ is a normalized source distribution $\left(S(z) / F_{h}\right)$ and $C_{n}$ is the portion of the concentration profile due to near-field effects.

The near-field resistance $r_{n}$ is not a measurable quantity, but rather a parameterization of the magnitude of the near-field effect on concentration-flux relationships. We mention it here because it is possible to determine it for the present model thus allowing a simple quantitative comparison of LNF and the present theory. Following Van Den Hurk and McNaughton (1995) and Raupach (1989), we created a range of ideal normalized source distributions $\phi(z)$ using a beta distribution function and profiles of normalized $\sigma_{w}$ and $L_{L}$ from the equations

$$
\begin{aligned}
\phi(z) & =\frac{1}{\beta(p, q)} z^{(p-1)}(1-z)^{(q-1)} \\
\frac{\sigma_{w}}{u_{*}} & =\left\{\begin{array}{cc}
c_{1} & \text { for } z / h>1 \\
c_{0}+\left(c_{1}-c_{0}\right) z / h & \text { for } z / h \leqslant 1
\end{array}\right. \\
\frac{\tau_{L} u_{*}}{h} & =\max \left[c_{2}, \frac{k(z-d)}{c_{1}^{2} h}\right] .
\end{aligned}
$$

From this we calculated the normalized resistance $r_{n} u_{*}$ for the present model by determining the concentration profiles and subtracting the far-field component (calculated by setting the exponential term in Equation (21) to 0 ) to determine $C_{n}$. The results are presented in Table I, which also includes the results from Van Den Hurk and McNaughton's (1995) Table I.

The present model is clearly much more sensitive to changes in the source distribution and turbulence profiles. Table I shows that, in general, the near-field effect is much stronger in the present model, in agreement with the previous assessment that LNF underestimates the near-field component. However, when the $\sigma_{w}$ profile 


\section{TABLE I}

Values of the normalised assistance $r_{n} u_{*}$ based on LNF and the present model. Results are shown for various values of $\sigma_{w}$ within the canopy, expressed by the parameter $c_{0}$ and for different source distributions, as controlled by the $p$ and $q$ parameters of the beta distribution function.

\begin{tabular}{llllll}
\hline Beta function parameters & $(p, q)$ & $c_{0}=0$ & $c_{0}=0.25$ & $c_{0}=0.5$ & $c_{0}=1.25$ \\
\hline LNF: & 2,4 & 0.43 & 0.42 & 0.41 & 0.38 \\
& 2,2 & 0.32 & 0.31 & 0.31 & 0.30 \\
& 4,2 & 0.36 & 0.36 & 0.35 & 0.33 \\
& 6,2 & 0.41 & 0.41 & 0.40 & 0.38 \\
\hline Present model: & 2,4 & 2.20 & 0.49 & 0.24 & 0.05 \\
& 2,2 & 1.36 & 0.49 & 0.30 & 0.11 \\
& 4,2 & 0.92 & 0.59 & 0.46 & 0.27 \\
& 6,2 & 0.88 & 0.64 & 0.53 & 0.35 \\
\hline
\end{tabular}

is constant through the canopy $\left(c_{0}=1.25\right)$, the near-field effect is much smaller than in LNF. The greater sensitivity of $r_{n}$ to changes in the parameters using our model is due to the near-field effect being a factor of the length scales at both source and gradient height, whereas LNF uses only the statistics at source height in determining the $C_{n}$ profile. Though $r_{n}$ is generally a relatively small term relative to the other resistances in a canopy, this analysis shows the importance of correctly determining the extent of the near-field effect before using the $r_{n}$ parameterization in a two-layer model.

\section{Discussion and Conclusions}

The preceding has shown the possibility of analytically relating scalar fluxes and profiles within a plant canopy. The model is able to do so by accounting for the Lagrangian nature of turbulent dispersion. Note that this model is independent of the turbulence structure, and is only a function of the Lagrangian nature of turbulent dispersion. In other words, what is significant is not the effect of the canopy on the air flow, but rather the proximity to the source that creates the persistence effects that this model accounts for. Therefore, as it stands, this model applies to any one-dimensional situation where the concentration profile near to the source is of concern.

In the case of a surface source the model reduces to

$$
\operatorname{Flux}_{i}=-\left.\sigma_{w i} L_{L i} \frac{d c}{d z}\right|_{i}
$$


implying that the flux from a surface source can be determined from measurements of the gradient, $\sigma_{w}$ and $L_{L}$ at any height. As shown in Section 4 it is possible to substitute $L_{E}$ for $L_{L}$, though further investigation of the relationship between the two under field conditions is necessary. This is a significant result because it means that for gases such as $\mathrm{N}_{2} \mathrm{O}$ and $\mathrm{CH}_{4}$, which are emitted from soils but do not interact with foliage, flux measurements using gradients are possible within a canopy near the surface. In addition to the benefit of increased resolution due to the much steeper gradients found near the surface, this also means that smaller areas will be incorporated into the flux calculation. This opens the way for more precise micrometeorological measurements of the spatial variability of emissions. This might also be applied to studies of fluxes from complex terrain, where long uniform fetches are not available.

Field tests of this model are still required. The authors are presently unaware of any field data sets with the requisite measurements for careful testing of our model. The success with the wind-tunnel data is encouraging, and previous studies have shown that wind-tunnel experiments do provide an accurate portrait of field situations (e.g., Novak et al., 2000).

The success of the model depends largely on the validity of applying a homogeneous solution to the inhomogeneity of canopy turbulence. Evidence that this is possible was provided by Raupach (1987). How far this can be extended to other inhomogeneous flows is not known, nor do we know if this assumption is applicable in all canopies. It seems likely that sparser canopies, such as the windtunnel canopy discussed above (frontal area index $=0.23$ ), will be suitable, but it is possible that very dense canopies would prove too inhomogeneous. It may also be that very stable or unstable conditions create prohibitive inhomogeneity. Further investigation of these questions under field conditions is again necessary.

\section{Acknowledgements}

We would like to thank the Natural Sciences and Engineering Research Council of Canada and the Atmospheric Environment Service for their generous support of this work. We would also like to thank the anonymous reviewers for their comments.

\section{References}

Collineau, S. and Brunet, Y.: 1993a, 'Detection of Turbulent Coherent Motions in a Forest Canopy, Part I: Wavelet Analysis', Boundary-Layer Meteorol. 65, 357-379.

Collineau, S. and Brunet, Y.: 1993b, 'Detection of Turbulent Coherent Motions in a Forest Canopy Part II: Time-Scales and Conditional Analysis', Boundary-Layer Meteorol. 66, 49-73.

Coppin, P. A., Raupach, M. R., and Legg, B. J.: 1986, 'Experiments on Scalar Dispersion within a Model Plant Canopy Part II: An Elevated Plane Source', Boundary-Layer Meteorol. 335, $167-191$. 
Corrsin, S.: 1963, 'Estimates of the Relations between Eulerian and Lagrangian Scales in Large Reynolds Number Turbulence', J. Atmos. Sci. 20, 115-119.

Csanady, G. T.: 1973, Turbulent Diffusion in the Environment, D. Reidel Publishing Company, Boston, MA, 248 pp.

Frenkiel, F. N.: 1953, 'Turbulent Diffusion: Mean Concentration Distribution in a Flow Field of Homogeneous Turbulence', Adv. Appl. Mech. 3, 61-107.

Kraichnan, R. H.: 1964, 'Relation between Lagrangian and Eulerian Correlation Times of a Turbulent Velocity Field', Phys. Fluids 7, 142-143.

Leclerc, M. Y. and Thurtell, G. W.: 1990, 'Footprint Prediction of Scalar Fluxes Using a Markovian Analysis', Boundary-Layer Meteorol. 52, 247-258.

McNaughton, K. G. and Van Den Hurk, B. J. J. M.: 1995, “A "Lagrangian” Revision of the Resistors in the Two-Layer Model for Calculating the Energy Budget of a Plant Canopy', Boundary-Layer Meteorol. 74, 261-288.

Novak, M. D., Warland, J. S., Orchansky, A. L., Ketler, R., and Green, S.: 2000, 'Wind Tunnel and Field Measurements of Turbulent Flow in Forests. Part I: Uniformly Thinned Stands', BoundaryLayer Meteorol. 95, 457-495.

Raupach, M. R.: 1987, ‘A Lagrangian Analysis of Scalar Transfer in Vegetation Canopies', Quart. J. Roy. Meteorol. Soc. 113, 107-120.

Raupach, M. R.: 1989, 'A Practical Lagrangian Method for Relating Scalar Concentrations to Source Distributions in Vegetation Canopies', Quart. J. Roy. Meteorol. Soc. 115, 609-632.

Raupach, M. R., Coppin, P. A., and Legg, B. J. 1986, 'Experiments on Scalar Dispersion within a Model Plant Canopy. Part I: The Turbulence Structure', Boundary-Layer Meteorol. 35, 21-52.

Raupach, M. R., Finnigan, J. J., and Brunet, Y.: 1989, 'Coherent Eddies in Vegetation Canopies', in Proc. Fourth Australasian Conf. on Heat and Mass Transfer, pp. 75-90.

Raupach, M. R., Finnigan, J. J., and Brunet, Y.: 1996, 'Coherent Eddies and Turbulence in Vegetation Canopies: The Mixing-Layer Analogy', Boundary-Layer Meteorol. 78, 351-382.

Richardson, L. F.: 1920, 'Some Measurements of Atmospheric Turbulence', Phil. Trans. Roy. Soc. Lond. A221, 1-28.

Rodean, H. C.: 1996, Stochastic Lagrangian Models of Turbulent Diffusion, Meteorological Monographs, Vol. 26, No. 48, American Meteorological Society, Boston, MA, 84 pp.

Shaw, R. H., Brunet, Y., Finnigan, J. J., and Raupach, M. R.: 1995, 'A Wind Tunnel Study of Air Flow in Waving Wheat: Two-Point Velocity Statistics', Boundary-Layer Meteorol. 76, 349-376.

Taylor, G. I.: 1915, 'Eddy Motions in the Atmosphere', Phil. Trans. Roy. Soc. Lond. A215, 1-26.

Taylor, G. I.: 1921, 'Diffusion by Continuous Movements', Proc. Lond. Math. Soc., Ser. 2 20, 196-211.

Van Den Hurk, B. J. J. M. and McNaughton, K. G.: 1995, 'Implementation of Near-Field Dispersion in a Simple Two-Layer Surface Resistance Model', J. Hydrol. 166, 293-311.

Wygnanski, I. and Fiedler, H. E.: 1970, 'The Two-Dimensional Mixing Region', J. Fluid Mech. 41, 327-361. 
